University of Wollongong

Research Online

Australian Institute for Innovative Materials -

Papers

Australian Institute for Innovative Materials

$1-1-2016$

\title{
Tuning superconductivity in FeSe thin films via magnesium doping
}

Wenbin Qiu

University of Wollongong,wq118@uowmail.edu.au

Zongqing $\mathrm{Ma}$

University of Wollongong, zqma@uow.edu.au

Yongchang Liu

Tianjin University

Md Shahriar Hossain

University of Wollongong, shahriar@uow.edu.au

Xiaolin Wang

University of Wollongong, xiaolin@uow.edu.au

See next page for additional authors

Follow this and additional works at: https://ro.uow.edu.au/aiimpapers

Part of the Engineering Commons, and the Physical Sciences and Mathematics Commons

Research Online is the open access institutional repository for the University of Wollongong. For further information contact the UOW Library: research-pubs@uow.edu.au 


\title{
Tuning superconductivity in FeSe thin films via magnesium doping
}

\begin{abstract}
In contrast to its bulk crystal, the FeSe thin film or layer exhibits better superconductivity performance, which recently attracted much interest in its fundamental research as well as in potential applications around the world. In the present work, tuning superconductivity in FeSe thin films was achieved by magnesium-doping technique. Tc is significantly enhanced from $10.7 \mathrm{~K}$ in pure FeSe films to $13.4 \mathrm{~K}$ in optimized Mg-doped ones, which is approximately 1.5 times higher than that of bulk crystals. This is the first time achieving the enhancement of superconducting transition temperature in FeSe thin films with practical thickness $(120 \mathrm{~nm}$ ) via a simple Mg-doping process. Moreover, these Mg-doped FeSe films are quite stable in atmosphere with $\mathrm{Hc} 2$ up to $32.7 \mathrm{~T}$ and Tc zero up to $12 \mathrm{~K}$, respectively, implying their outstanding potential for practical applications in high magnetic fields. It was found that Mg enters the matrix of FeSe lattice, and does not react with FeSe forming any other secondary phase. Actually, Mg first occupies Fe-vacancies, and then substitutes for some Fe in the FeSe crystal lattices when Fe-vacancies are fully filled. Simultaneously, external Mg-doping introduces sufficient electron doping and induces the variation of electron carrier concentration according to Hall coefficient measurements. This is responsible for the evolution of superconducting performance in FeSe thin films. Our results provide a new strategy to improve the superconductivity of 11 type Fe-based superconductors and will help us to understand the intrinsic mechanism of this unconventional superconducting system.
\end{abstract}

\section{Keywords}

fese, superconductivity, thin, magnesium, tuning, doping, films, via

Disciplines

Engineering | Physical Sciences and Mathematics

\section{Publication Details}

Qiu, W., Ma, Z., Liu, Y., Hossain, M. Shahriar Al., Wang, X., Cai, C. \& Dou, S. Xue. (2016). Tuning superconductivity in FeSe thin films via magnesium doping. ACS Applied Materials and Interfaces, 8 (12), 7891-7896.

\section{Authors}

Wenbin Qiu, Zongqing Ma, Yongchang Liu, Md Shahriar Hossain, Xiaolin Wang, Chuanbing Cai, and S X. Dou 


\title{
Tuning superconductivity in FeSe thin films via Magnesium doping
}

\author{
Wenbin $\mathrm{Qiu}^{2,3}$, Zongqing $\mathrm{Ma}^{1,2 *}$, Yongchang $\mathrm{Liu}^{1}$, Mohammed Shahriar Al Hossain ${ }^{2}$, Xiaolin Wang ${ }^{2}$, \\ Chuanbing Cai ${ }^{3}$, Shi Xue Dou ${ }^{2}$ \\ ${ }^{1}$-Tianjin Key Laboratory of Composite and Functional Materials, School of Materials Science \& \\ Engineering, Tianjin University, Tianjin 300072, People's Republic of China \\ ${ }^{2}$-Institute for Superconducting and Electronic Materials, AIIM, University of Wollongong, Squires Way, North \\ Wollongong, NSW 2500, Australia \\ ${ }^{3}$-Shanghai Key Laboratory of High Temperature Superconductors, Physics Department, Shanghai University, \\ Shanghai 200444, People's Republic of China
}

\begin{abstract}
:
In contrast to its bulk crystal, the FeSe thin film or layer exhibits better superconductivity performance, which recently attract much interest in its fundamental research as well as potential application around the world. In present work, tuning superconductivity in FeSe thin films was achieved by magnesium-doping technique. $T_{c}$ is significantly enhanced from $10.7 \mathrm{~K}$ in pure FeSe films to $13.4 \mathrm{~K}$ in optimized $\mathrm{Mg}$-doped ones, which is approximately 1.5 times higher than that of bulk crystals. This is the first time to achieve the enhancement of superconducting transition temperature in FeSe thin films with practical thickness $(120 \mathrm{~nm})$ via a simple Mg-doping process. Moreover, these Mg-doped FeSe films are quite stable in atmosphere with $H_{c 2}$ up to $32.7 \mathrm{~T}$ and $T_{c}^{\text {zero }}$ up to $12 \mathrm{~K}$, respectively, implying their outstanding potential for practical applications in high magnetic fields. It was found that $\mathrm{Mg}$ enters the matrix of FeSe lattice, not react with FeSe forming any other secondary phase. Actually, Mg first occupies Fe-vacancy, and then substitutes for some Fe in the FeSe crystal lattices when Fe-vacancies are fully filled.
\end{abstract}

\footnotetext{
* Corresponding author E-mail: mzq0320@163.com
} 
Simultaneously, external Mg-doping introduces sufficient electron doping and induces the variation of electron carrier concentration according to Hall coefficient measurements. This is responsible for the evolution of superconducting performance in FeSe thin films. Our results provide a new strategy to improve the superconductivity of 11 type Fe-based superconductors and will help to understand the intrinsic mechanism of this unconventional superconducting system.

Keywords: FeSe film; superconductivity; pulsed laser deposition; $\mathrm{Mg}$ doping; electron concentration; 


\section{Introduction}

The discovery of iron-based materials with a high superconducting transition temperature $T_{c}$ has attracted much attention for both fundamental studies and practical applications ${ }^{1}$. Till now, plenty of efforts have been made to obtain higher superconducting performance, but the intrinsic mechanism of superconductivity in these systems is still being explored. Among iron-based superconducting family, the Iron selenide (FeSe) has the simplest structure with binary chemical composition, consisting of only two-dimensional conducting planes ${ }^{2}$, rendering it to be an appropriate candidate for exploring the intrinsic mechanism of iron-based superconductors. Compared with its bulk crystals, FeSe in the form of film or multilayer attracts more attention as large enhancement in superconducting performance can be obtained. Especially, high $T_{c}$ (around $77 \mathrm{~K}$ ) was reported in single unit-cell FeSe layer ${ }^{3}$, which is considered as the highest value among all the iron-based superconductors. As for the enhancement mechanism of superconductivity in FeSe film or layers, there is still a hot debate, and more fundamental work is still required to understand the superconductivity of FeSe films and multilayer in depth. On the other hand, the instability of FeSe single layer or multilayer in atmosphere serves as the largest obstacle from further various characteristics or physical measurements to explore the nature of superconductivity in FeSe system. Hence, with the atmosphere-stable property, highperformance FeSe thin film is more suitable for fundamental research and practical application as well.

Recently, we have succeeded in enhancing superconductivity in FeSe thin films by tuning the Fevacancy disorders. The amount of Fe-vacancy disorders can effectively control the electron carrier concentration, determining superconductor-insulator transition (SIT) and the evolution of superconductivity in FeSe thin films. Actually, controlling the carrier concentration is one of the 
most effective ways to acquire better superconductivity in iron-based superconductors. Till now, the regulation of charge carrier can be realized via various methods, such as adjusting stoichiometric composition ${ }^{4-6}$, chemical doping ${ }^{7-9}$, liquid-gating technique ${ }^{10}$, etc. Understanding the nature of carrier concentration variation in FeSe film can provide a valuable hint to reveal the intrinsic mechanism of superconductivity in this system.

In present work, tuning the superconductivity of FeSe thin films was achieved by $\mathrm{Mg}$ doping technique. Distinct enhancement in $T_{c}$ is obtained from $10.7 \mathrm{~K}$ in pure FeSe film to $13.4 \mathrm{~K}$ in optimized Mg-doped ones. Mg-doping can introduce electron doping and induce the variation of electron carrier concentration, which significantly influences ultimate superconducting performance in FeSe films. With increasing the content of Mg-doping, a dome-shaped $\mathrm{Mg}$ content dependence of $T_{c}$ is observed for the first time. Once certain content of Mg-doping is reached, one slump in $T_{c}$ occurs together with obvious variation in both the XRD diffraction peak position and hall coefficient, implying nearly vanishing of superconductivity. It is believed that moderate Mg-doping has positive effect on the superconducting performance in FeSe films through the introduction of sufficient electron doping, but degradation or even collapse will occur to superconductivity when excessive content of $\mathrm{Mg}$ is introduced into FeSe films.

\section{Experimental}

FeSe films are grown on $\mathrm{CaF}_{2}(100)$ single crystal (lattice parameter $a=5.462 \AA$ ) by PLD technique (Nd: YAG, $355 \mathrm{~nm}, 10 \mathrm{~Hz}, 2 \mathrm{~W}$ output). The substrate temperature is chosen as $300{ }^{\circ} \mathrm{C}$

and the entire experiment is carried out in a closed chamber with vacuum better than $5 \times 10^{-4} \mathrm{~Pa}$. Home-made FeSe pellet which is prepared from commercial available Fe and Se powder serves as the target for FeSe sputtering. In order to check the influence of Mg content on FeSe films, the 
thickness of FeSe films is strictly fixed at $120 \mathrm{~nm}$ which shows ordinary $T_{c}$ as $10.7 \mathrm{~K}$ in our previous work ${ }^{4}$. Right after the deposition of FeSe film, Mg-deposition is introduced by directly switching to $\mathrm{Mg}$ target holder. By controlling the deposition time of $\mathrm{Mg}$ as $1.5 \mathrm{~min}, 3 \mathrm{~min}, 10$ min and 20 min, a batch of Mg-doped FeSe films are obtained and denoted as \#FM0 (pure FeSe), \#FM1, \#FM2, \#FM3 and \#FM4, respectively. Both of the electrical resistivity and Hall coefficient measurements are carried out in a $9 \mathrm{~T}$ physical property measurement system (PPMS). XRD $\theta-2 \theta$ scan is used to characterize the crystal structure of films. For checking the surface morphology and chemical composition, scanning electron microscopy (SEM, JEOL JSM-6490LV) and high-accuracy energy dispersive X-ray spectrum (EDX) detector (Oxford Instruments $\mathrm{X}$-MaxN 80) is employed.

\section{Results and discussion}

Fig. 1(a) shows the temperature dependence of electrical resistivity for all films (\#FM0, \#FM1, \#FM2, \#FM3, and \#FM4) in the magnified temperature range while full range up to $300 \mathrm{~K}$ is plotted in the inset of Fig. 1(a). The $\rho(\mathrm{T})$ curves with positive slope are exhibited in all of them, indicating classical metallic behaviors. At normal state, the resistivity of FeSe films keeps at low level owing to the good conductivity after introducing small amount of Mg. Nevertheless, it dramatically increases in excessive Mg-doped sample \#FM4 probably because of the severe scattering effect of electrons, which is introduced by excessive Mg-doping. In order to recognize the change of $T_{c}$ clearly, $\mathrm{d}\left(\mathrm{R} / \mathrm{R}_{300 \mathrm{~K}}\right) / \mathrm{dT}$ curves are illustrated in Fig. 1(b). In pure FeSe film \#FM0, resistivity drops sharply at $T_{c}^{\text {onset }} \approx 10.7 \mathrm{~K}$ and reaches zero at $T_{c}^{\text {zero }} \approx 9.8 \mathrm{~K}$. With the

introducing of Mg-doping, $T_{c}^{\text {onset }}$ increases systematically and the highest $T_{c}{ }^{\text {onset }} \approx 13.4 \mathrm{~K}$ is achieved in optimized Mg-doped sample \#FM2, which is approximately 1.5 times higher than 
that of bulk crystals. Further increasing the content of $\mathrm{Mg}$-doping, degradation in $T_{c}{ }^{\text {onset }}$ is observed in \#FM3 but $T_{c}^{\text {onset }}$ still remains higher than that in un-doped \#FM0. Interestingly, an incomplete transition down to $4.2 \mathrm{~K}$ is detected in over-doped sample \#FM4. The possible explanation will be given below combined with other characterizations including XRD and Hall measurement. According to the $T_{c}$ results above, our work is the first time to achieve enhancement of superconducting transition temperature in atmosphere-stable FeSe thin films with practical thickness $(120 \mathrm{~nm})$ via a simple $\mathrm{Mg}$-doping process. Despite current $T_{c}$ is not very high compared with the result of Te substitution or ultra-thin technique ${ }^{3,11,12}$, what we explored here is a new inspiring way to improve the superconductivity of traditional FeSe films.

The temperature dependence of the upper critical magnetic field $H_{c 2}(\mathrm{~T})$ is plotted in Fig. 1(c) and the linear extrapolations to $\mathrm{T}=0 \mathrm{~K}$ are shown in the inset. $H_{c 2}$ determined as a function of $T_{c}{ }^{\text {mid }}$. $H_{c 2}$ of \#FM4 is not shown here because of the incompleteness of transition. The estimated $H_{c 2}$ in other four samples are about 27.8 T, 31.2 T, 32.7 T and 29.1 T for \#FM0, \#FM1, \#FM2, \#FM3 and \#FM4 respectively. One can see that Mg-doped FeSe films exhibit higher $H_{c 2}$ than un-doped one, and the best $H_{c 2}$ value as high as $32.7 \mathrm{~T}$ is obtained in \#FM2, exhibiting great potential of our atmosphere-stable FeSe superconducting films for high-field applications.

As shown above, improvement in superconductivity of FeSe thin films can be achieved by Mgdoping, it is of great importance to understand its intrinsic enhancement mechanism. It is well known that charge carrier concentration is one of the most crucial parameters that influence the superconductivity in iron-based superconductors ${ }^{13}$. As Mg belongs to the group of alkaline earth, abundant electron carriers are supposed to be provided through doping. Hereby hall measurements are performed to reveal the state of charge carrier in all 5 samples. In Fig. 2, temperature dependences of Hall coefficient $\left(R_{H}\right)$ are illustrated. $R_{H}$ is defined as $R_{H}=\rho_{x y} / B$, 
where $\rho_{\mathrm{xy}}$ stands for Hall transverse resistivity and B is designated field under fixed temperatures ranging from $20 \mathrm{~K}$ to $300 \mathrm{~K} . \mathrm{R}_{\mathrm{H}}$ in all the samples have negative value at the temperature above $T_{c}$, indicating that electrons are dominated carrier type in all of our $\mathrm{Mg}$-doped FeSe films. From room temperature to $150 \mathrm{~K}$, the $\mathrm{R}_{\mathrm{H}}$ of all 5 samples is almost temperature independent. Below that, the absolute value of $\mathrm{R}_{\mathrm{H}}$ starts to increase, implying the multiband nature in typical FeSe system. Carrier concentration ( $n$ ) indicates the amount of carriers in unit volume. The relation between $\mathrm{R}_{\mathrm{H}}$ and $n$ is deduced in equation (1):

$$
R_{H}=\frac{E_{y}}{j_{x} B}=\frac{V_{H} t}{I B}=-\frac{1}{n e}
$$

$\left(\boldsymbol{R}_{\boldsymbol{H}}\right.$ - Hall coefficient, $\boldsymbol{E}_{\boldsymbol{y}}$ - induced electric field, $\boldsymbol{j}$ - the current density of the carrier electrons, $\boldsymbol{B}$ - magnetic field, $\boldsymbol{V}_{\boldsymbol{H}}$ - Hall voltage, $\boldsymbol{t}$ - the thickness of the plate, $\boldsymbol{I}$ - the current across the plate length, $\boldsymbol{e}$ - elementary charge, $\boldsymbol{n}$ - carrier concentration). As carrier concentration $(n)$ is inversely proportional to $\mathrm{R}_{\mathrm{H}}$, the increasing $\mathrm{R}_{\mathrm{H}}$ in minus side at lower temperature region represents that $n$ decreases with lowering temperature. Similar Temperature dependence of $R_{H}$ is observed in undoped \#FM0, lightly-doped \#FM1, \#FM2 and heavily-doped \#FM3. However, large absolute value of $R_{H}$ is obtained in over-doped \#FM4, suggesting a severe reduction in electron concentration. It can be considered that excessive Mg-doping is responsible for the extremely low electron concentration. It seems that the transmission property of electronic state is significantly changed for some reason, which is consistent with the decaying behavior in the temperature dependence of resistivity, as shown in Fig. 1(a). Hence, by showing the collapse in carrier concentration, the investigation in Hall coefficient offers a strong evidence for the degradation of superconductivity in \#FM4.

In order to distinguish the tendency of \#FM0, \#FM1, \#FM2 and \#FM3, magnified view of $R_{H}$ in low-temperature region is shown in the inset of Fig. 2. Interestingly, an abnormal lower electron 
concentration is found below $80 \mathrm{~K}$ in lightly-doped \#FM1 which shows significantly enhanced superconductivity. It might result from a competitive mechanism between "self-doping" and "external Mg-doping" in \#FM1. Actually, in our un-doped FeSe films, Fe-vacancy disorder induced "self-doping" exists. The discovery of disorder-induced "self-doping" is raised by T. Berlijn's group ${ }^{14}$ in the system of $\mathrm{KFe}_{2} \mathrm{Se}_{2}$. By angle-resolved photoelectron spectroscopy (ARPES), they found that Fe-vacancy disorder can provide significant electron concentration $\left(n_{e}\right)$. According to the analysis in our previous work ${ }^{4}$, similar increment in $n_{e}$ is detected in pure FeSe films in which Fe-vacancy disorders are detected. This is the reason why un-doped FeSe film (\#FM0) still shows relatively high $\mathrm{R}_{\mathrm{H}}$ value.

Once Mg is introduced into FeSe films, "external Mg-doping" starts to affect the electron state of FeSe together with original "self-doping". It is speculated that there is one "mixed-doping state" exists when two doping modes are comparable to each other. In lightly-doped sample (\#FM1), only very little $\mathrm{Mg}$ is implanted into FeSe layers. In this case, doping performance will be offset or even produce lower $n_{e}$ than un-doped sample (\#FM0). But, improvement of superconductivity is still observed in lightly-doped sample (\#FM1). It might be because Fe-vacancy is occupied by Mg that enters FeSe lattice, which will be discussed in detail later combined with XRD results. With further increasing content of external Mg-doping, the effect of "self-doping" becomes ignorable whilst big amount of electron carrier is introduced from $\mathrm{Mg}$ into FeSe films, reflecting by the increment of $\left|R_{H}\right|$ in the results of Hall coefficient. As a result, the "external-doping" become dominating in providing charge carrier rather than "self-doping" in \#FM2 and \#FM3, but the intrinsic mechanism is still needed to be clarified in future work. It is noteworthy that the superconducting performance in \#FM3 is not as good as that in \#FM2, even though they possess the similar electron concentration. It is due to the decreased orientation degree of FeSe film in 
\#FM3, which will be discussed later based on XRD results. In the case of over-doped \#FM4, abrupt decay is found in electron state and superconducting performance. It is ascribed to both severe electron scattering and the destruction of superconducting structure derived from excessive Mg doping. Based on the results above, Mg-doping is demonstrated as an effective way to introduce significant electron carrier into FeSe films.

Typical XRD $\theta-2 \theta$ spectrums are illustrated in Fig. 3(a), ranging from 10 to 80 degree. One can see that highly (00l) oriented FeSe texture based on $\mathrm{PbO}$ structure exists in all 5 samples. Noteworthily, highly $\mathrm{c}$-axis oriented $\beta$-FeSe phases are observed. It is manifested that Mg enters FeSe lattice instead of reacting with FeSe to form new phases. This speculation is diverse from the result raised in $\mathrm{Mg}$-doped $\mathrm{FeSe}$ bulks ${ }^{8}$ where lattice parameters of FeSe remain unchanged and $\mathrm{MgSe}$ phase is formed with $\mathrm{Mg}$ addition, representing absolutely different doping mechanism of $\mathrm{Mg}$ in FeSe films and bulks. In Mg-doped FeSe bulks made by solid-state sintering, the small and reactive $\mathrm{Mg}$ particles are prone to react with FeSe particles. Subsequently, lots of secondary phases are generated at relatively higher sintering temperature. Oppositely, in present work, Mg-doping is introduced by low-temperature sputtering using PLD technique. It enables homogeneous distribution of $\mathrm{Mg}$ element within FeSe films. As a result, $\mathrm{Mg}$ can readily enter FeSe lattice, and bring about novel properties in this system. Moreover, in Fig. 3(a), it is also found that $\beta$-FeSe (101) diffraction peak appears in Mg heavily-doped (\#FM3) sample, which induces a negative effect on texture degree and could bring about deterioration on superconductivity, as shown in Fig. 1(a).

Fig. 3(b) shows the enlarged interval near $\beta$-FeSe (001) peak. Slight shift of diffraction peaks is observed in all Mg-doped FeSe films (\#FM1, \#FM2, \#FM3 and \#FM4) compared with un-doped one (\#FM0). The specific $2 \theta$ values are extracted and plotted in the inset of Fig. 3(b), where the 
dashed line represents the case of un-doped sample (\#FM0). It was recognized that $\beta$-FeSe (001) peak locates at lower $2 \theta$ in \#FM1, \#FM2 and \#FM3 than that in \#FM0, demonstrating the elongation in $c$-axis parameter after Mg-doping. On the contrary, the $\beta$-FeSe (001) peak of \#FM4 with excessive content of $\mathrm{Mg}$-doping is found to locate at higher $2 \theta$ angle, that is to say, the lattice along c-axis shrinks. The variation of diffraction peak position can be understood well combined with the effect of Mg-doping. It has been confirmed that plenty of Fe-vacancies exist in as-grown FeSe films ${ }^{15}$. During the diffusion of $\mathrm{Mg}$ element into FeSe films, Mg enters the FeSe crystal lattice and occupies Fe-vacancies so that the elongation of lattice parameter is generated. Simultaneously, additional electron carrier is introduced and consequently, higher superconductivity is acquired, as shown in Fig. 1 and Fig. 2. Further increasing the content of Mg-doping, some of the Fe sites in the FeSe superconducting lattice are likely to be replaced by excessive $\mathrm{Mg}$. As the $\mathrm{Mg}^{2+}$ is smaller than that of $\mathrm{Fe}^{2+}$ in radius, shrinkage in c-axis parameter is rational. Moreover, because of the substitution of $\mathrm{Mg}$ for $\mathrm{Fe}$, destruction of superconducting structure and severe electron scattering serve as negative factors which bring about the collapse in superconductivity. In over-doped sample (\#FM4), strong suppression in superconductivity is found, corresponding to the results in superconducting performance and Hall coefficient. Speculation can be easily made that the different content of Mg-doping drives the evolution of superconducting properties in all FeSe films. For acquiring how much Mg doping is introduced, EDX analysis is employed. It is a qualified candidate due to the adequate penetration depth as well as high accuracy for chemical composition analysis. In this work, we acquire element content of $\mathrm{Mg}, \mathrm{Fe}$ and $\mathrm{Se}$ throughout the entire film and calculate the atomic ratio of $\mathrm{Mg} / \mathrm{FeSe}$ to represent the implantation content of Mg-doping. Detailed information including the atomic ratio of $\mathrm{Mg} / \mathrm{FeSe}$, the carrier concentration at $30 \mathrm{~K}$, and superconducting performance of all 5 samples 
are listed in Table 1. These results will strongly support and give reference to our following discussions on the mechanism of Mg-doping effect.

Based on Table 1, the $T_{c}$ and relative electron concentration $\mathrm{n} / \mathrm{n}_{\mathrm{HFM} 3}$ at $30 \mathrm{~K}$ of all five samples are both presented in Fig. 4 as a function of the content of $\mathrm{Mg}$ doping. The content of $\mathrm{Mg}$ within \#FM0, \#FM1, \#FM2, \#FM3 and \#FM4 is $0 \%, 6.4 \%, 13.1 \%, 26.2 \%$ and $75.3 \%$ as detected. It is in approximate linear dependence with the increment of Mg-deposition time, demonstrating our reliable deposition technique in controlling yield proportionally. However, $T_{c}$ varies in a more complicated way. When little amount of $\mathrm{Mg}$ is introduced, despite that electron concentration is slightly reduced due to the "mixed-state" mentioned above, great improvement in $T_{c}$ by $2 \mathrm{~K}$ is detected in \#FM1. It is explained that Mg enters FeSe lattice and occupies Fevacancy so that defects introduced by Fe-vacancy disorder are eliminated and more ideal superconducting structure might be achieved. Further increasing the content of Mg-doping, "external Mg-doping" becomes the dominating factor rather than original Fe-vacancy disorder induced "self-doping". With heavy electron doping, $T_{c}$ up to $13.4 \mathrm{~K}$ is obtained in \#FM2, which has been enhanced by more than $25 \%$ compared with un-doped \#FM0. In the case of heavilydoped sample (\#FM3), $\beta$-FeSe (101) peak emerges in XRD pattern (see Fig. 1(a)). The reduction in orientation degree of FeSe films may lead to lower superconductivity even though the electron carrier concentration in this sample is comparable to that of \#FM2 with the highest $T_{c}$. As expected, degradation in $T_{c}$ and $\Delta T_{c}$ is detected in heavily-doped sample \#FM3. In the case of over-doped sample \#FM4, an abrupt drop in $T_{c}$ occurs as low as $4.9 \mathrm{~K}$. It should be ascribed to both the severe electron scattering and the destruction of superconducting structure owing to the result of substitution effect. Ultimately, a dome-shaped tendency is observed in the tuning effect of Mg-doping on $T_{c}$. It is noticed that the highest $T_{c}$ is acquired in the region with high electron 
concentration and the collapse of $T_{c}$ occurs when electron concentration remains at very low level. Hence, electronic state, especially electron carrier concentration is verified to possess significant impact on the superconductivity in FeSe films. In addition, owing to the advantage of simplicity and effectiveness, external Mg-deposition technique is highly expected as one promising way to provide controllable electron doping into FeSe films.

\section{Conclusion}

To summarize, we demonstrated that a simple Mg-doping procedure on as-grown FeSe thin film can effectively tune the electronic state and lattice structure of original FeSe. Enhancement of $T_{c}$ and $H_{c 2}$ are obtained up to $13.4 \mathrm{~K}$ and $32.7 \mathrm{~T}$, respectively under optimized content of $\mathrm{Mg}$ doping. According to Hall coefficient measurement, electron serves as the dominant type of charge carrier in both un-doped and Mg-doped FeSe films. It is found that small amount of Mgdoping can introduce significant electron concentration into FeSe films, in which better superconductivity is exhibited. However, abnormal reduction of electron concentration was found in FeSe film with excessive content of Mg-doping, which brings about the severe degradation in superconducting performance. Based on XRD results, highly (00l) oriented texture and slight shift of diffraction peaks are both observed in all Mg-doped FeSe films, indicating that $\mathrm{Mg}$ element enters the matrix of FeSe lattice, rather than reacts with FeSe and forming any other secondary phase. What's more, our Mg-doped FeSe films possess the property of atmosphere-stabilization which is a critical factor in the practical application. Our work is the first attempt to study the effect of heavy electron doping on traditional FeSe thin films via alkali earth metal doping, which not only provides a new strategy to improve the superconductivity of 11 type Fe-based films, but also helps to understand the intrinsic mechanism of this 
unconventional superconducting system.

Acknowledgement This work is supported by the Australian Research Council (Grant No. DE140101333). The authors are also grateful to the China National Funds for Distinguished Young Scientists (Grant No. 51325401) and National Natural Science Foundation of China (Grant No. 51302186 and 51574178). The authors acknowledge use of facilities within the UOW Electron Microscopy Centre. 


\section{References:}

(1) Kamihara, Y.; Watanabe, T.; Hirano, M.; Hosono, H. Iron-Based Layered Superconductor La $\left[\mathrm{O}_{1-\mathrm{x}} \mathrm{F}_{\mathrm{x}}\right]$ FeAs $(\mathrm{x}=0.05-0.12)$ with $T_{c}=26 \mathrm{~K}$. J. Am. Chem. Soc. 2008, 130, 3296-3297.

(2) Hsu, F. -C.; Luo, J. -Y.; Yeh, K. -W.; Chen, T. -K.; Huang, T. -W.; Wu, P. M.; Lee, Y. -C.; Huang, Y. -L.; Chu, Y. -Y.; Yan, D. -C.; Wu, M. -K. Superconductivity in the PbO-Type Structure $\alpha$-FeSe. Proc. Natl. Acad. Sci. U.S.A. 2008, 105, $14262-14264$.

(3) Wang, Q. -Y.; Li, Z.; Zhang, W. -H.; Zhang, Z. -C.; Zhang, J. -S.; Li, W.; Ding, H.; Ou, Y. -B.; Deng, P.; Chang, K.; Wen, J.; Song, C. -L.; He, K.; Jia, J. -F.; Ji, S. -H.; Wang, Y. -Y.; Wang, L. L.; Chen, X.; Ma, X. -C.; Xue, Q. -K. Interface-Induced High-Temperature Superconductivity in Single Unit-Cell FeSe Films on $\mathrm{SrTiO}_{3}$. Chin. Phys. Lett. 2012, 29, 037402.

(4) Qiu, W. -B.; Ma, Z. -Q.; Liu, Y. -C.; Wang X. -L.; Dou, S. -X. Transition from Insulator to Superconductor and Significant Enhancement of Superconductivity in FeSe Films via Tuning Fevacancy Disorders. arXiv:1512.00352.

(5) Stewart, G. -R.; Superconductivity in Iron Compounds. Rev. Mod. Phys. 2011, 83, 15891652.

(6) Wu, X. -J.; Zhang, Z. -Z.; Zhang, J. -Y.; Li, B. -H.; Ju, Z. -G.; Lu, Y. -M.; Li, B. -S.; Shen, D.

-Z. Two-carrier Transport and Ferromagnetism in FeSe Thin Films. J. Appl. Phys. 2008, 103, 113501.

(7) Liu, T. -J.; Ke, X.; Qian, B.; Hu, J.; Fobes, D.; Vehstedt, E. -K.; Pham, H.; Yang, J. -H.; Fang, M. -H.; Spinu, L.; Schiffer, P.; Liu, Y.; Zao, Z. -Q. Charge-carrier Localization Induced by Excess Fe in the Superconductor $\mathrm{Fe}_{1+\mathrm{y}} \mathrm{Te}_{1-\mathrm{x}} \mathrm{Se}_{\mathrm{x}}$. Phys. Rev. B 2009, 80, 174509.

(8) Lan, F.; Ma, Z. -Q.; Liu, Y. -C.; Chen, N.; Cai, Q.; Li, H. -J.; Barua, S.; Patel, D.; Hossain, M. -S. -A.; Kim, J. -H.; Dou, S. -X. The Formation of Nano-Layered Grains and Their Enhanced Superconducting Transition Temperature in Mg-doped FeSe ${ }_{0.9}$ Bulks. Sci. Rep. 2014, 4, 6481. 
(9) Miyata, Y.; Nakayama, K.; Sugawara, K.; Sato, T.; Takahashi, T. High-Temperature Superconductivity in Potassium-Coated Multilayer FeSe Thin Films. Nat nat..Mater. 2015, 14, $775-780$.

(10) Lei, B.; Cui, J. -H.; Xiang, Z. -J.; Shang, C.; Wang, N. -Z.; Ye, G. -J.; Luo, X. -G.; Wu, T.; Sun, Z:; Chen, X. H. Evolution of High-Temperature Superconductivity from Low- $T_{c}$ Phase Tuned by Carrier Concentration in FeSe Thin Flakes. Phys. Rev. Lett. 2016, 116, 077002.

(11) Tan, S. -Y.; Zhang, Y.; Xia, M.; Ye, Z. -R.; Chen, F.; Xie, X.; Peng, R.; Xu, D. -F.; Fan, Q.; Xu, H. -C.; Jiang, J.; Zhang, T.; Lai, X. -C.; Xiang, T.; Hu, J. -P.; Xie B. -P.; Feng, D. L. Interface-Induced Superconductivity and Strain-Dependent Spin Density Waves in FeSe/SrTiO 3 Thin Films. Nat. Mater. 2013, 12, 634-640.

(12) Zhang, W. -H.; Li, Z.; Li, F. -S.; Zhang, H. -M.; Peng, J. -P.; Tang, C. -J.; Wang, Q. -Y.; He, K.; Chen, X.; Wang, L. -L.; Ma, X. -C.; Xue, Q. -K. Interface Charge Doping Effects on Superconductivity of Single-Unit-Cell FeSe Films on $\mathrm{SrTiO}_{3}$ Substrates. Phys. Rev. B 2014, 89, $060506(\mathrm{R})$.

(13) Yeh, K. -W.; Huang, T. -W.; Huang, Y. -L.; Chen, T. -K.; Hsu, F. -C.; Wu, P. M.; Lee, Y. -C.; Chu, Y. -Y.; Chen, C. -L.; Luo, J. -Y.; Yan D. -C.; Wu, M. -K. Tellurium Substitution Effect on Superconductivity of the $\alpha$-Phase Iron Selenide. EPL, 2008, 84, 37002.

(14) Berlijn, T.; Hirschfeld, P. J.; Ku, W. Effective Doping and Suppression of Fermi Surface Reconstruction via Fe Vacancy Disorder in $\mathrm{K}_{\mathrm{x}} \mathrm{Fe}_{2-\mathrm{y}} \mathrm{Se}_{2}$. Phys. Rev. Lett. 2012, 109, 147003.

(15) Chen, T. -K.; Chang, C. -C.; Chang, H. -H.; Fang, A. -H.; Wang, C. -H.; Chao, W. -H.; Tseng, C. -M.; Lee, Y. -C.; Wu, Y. -R.; Wen, M. -H.; Tang, H. -Y.; Chen, F. -R.; Wang, M. -J.; Wu, M. -K.; Dyck, D. V. Fe-Vacancy Order and Superconductivity in Tetragonal $\beta$-Fe ${ }_{1-x}$ Se. Proc. Natl. Acad. Sci. U.S.A. 2014, 111, 63-68. 
Table 1. Detailed information of all prepared samples.

\begin{tabular}{|c|c|c|c|c|c|c|}
\hline & $\begin{array}{c}\text { Mg/FeSe } \\
(\%)\end{array}$ & $\begin{array}{c}\text { n at } 30 \mathrm{~K} \\
\left(\times 10^{20} \mathrm{~cm}^{-3}\right)\end{array}$ & $\begin{array}{c}\mathbf{n} / \mathbf{n}_{\# \text { FM3 }} \\
\text { at } 30 \mathrm{~K} \\
(\%)\end{array}$ & $\begin{array}{c}T_{c}^{\text {onset }} \\
\text { (K) }\end{array}$ & $\begin{array}{l}\Delta T_{c} \\
(K)\end{array}$ & $\begin{array}{r}H_{c 2} \\
(\mathbf{T})\end{array}$ \\
\hline \#FM0 & 0 & 8.2 & 80.4 & 10.7 & 1.9 & 27.8 \\
\hline \#FM1 & 6.4 & 7.8 & 71.6 & 12.7 & 1.2 & 31.2 \\
\hline \#FM2 & 13.1 & 10.6 & 97.4 & 13.4 & 1.4 & 32.7 \\
\hline \#FM3 & 26.2 & 10.9 & 100 & 11.4 & 1.8 & 29.1 \\
\hline \#FM4 & 75.3 & 2.3 & 21.3 & 4.9 & $\mathrm{n} / \mathrm{a}$ & $\mathrm{n} / \mathrm{a}$ \\
\hline
\end{tabular}


Figure 1
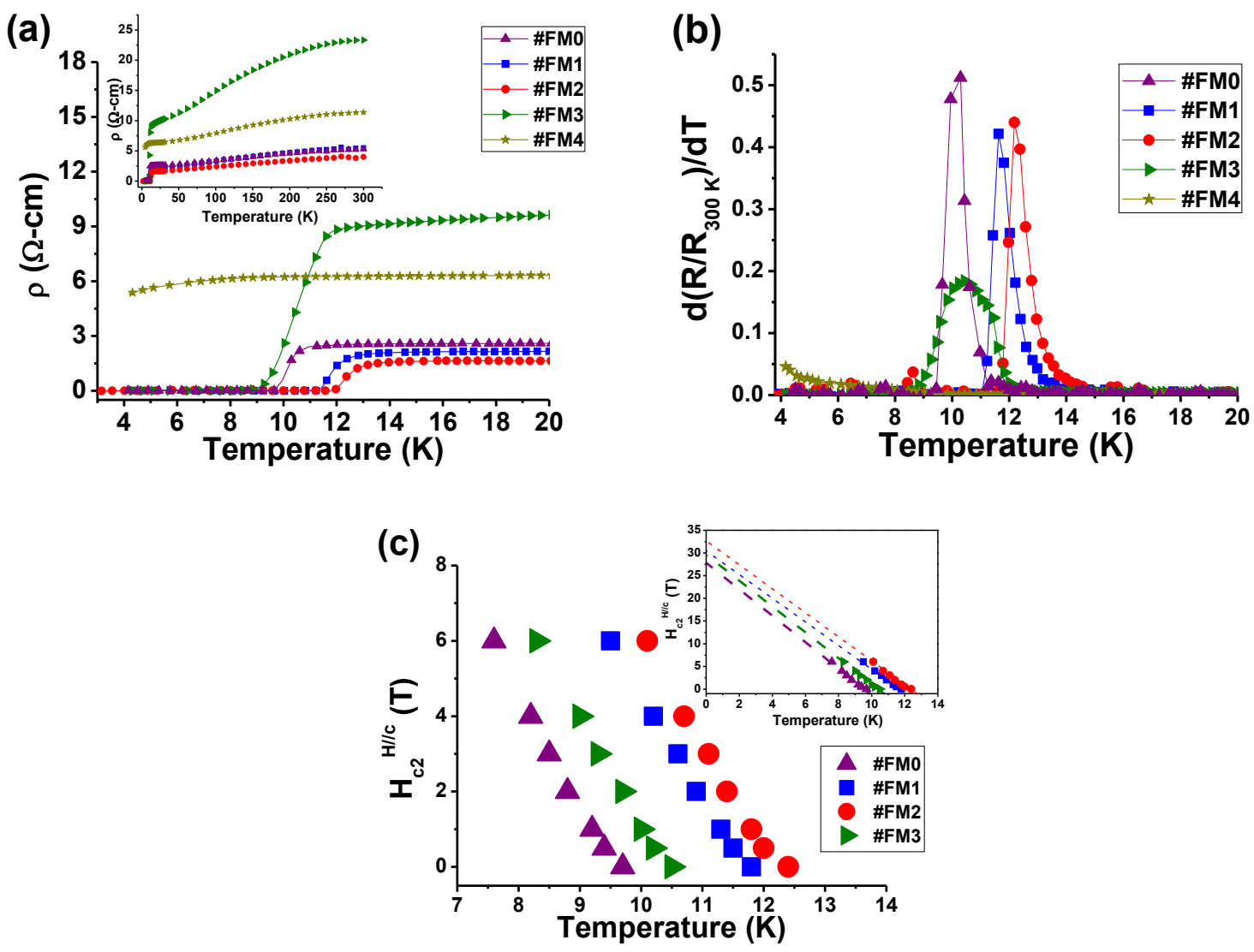

FIG. 1. (a) Temperature dependences of resistivity (R-T curves) at self-field for Mg-doped FeSe films with different deposition time of $\mathrm{Mg}(0 \mathrm{~min}, 1.5 \mathrm{~min}, 3 \mathrm{~min}, 10 \mathrm{~min}$ and $20 \mathrm{~min}$ ) ranging from $4 \mathrm{~K}$ to $20 \mathrm{~K}$. Inset is the full range R-T data from room temperature to $4.2 \mathrm{~K}$. (b) $d\left(R / R_{300 \mathrm{~K}}\right) / \mathrm{dT}$ curves for all five films from 4 to $20 \mathrm{~K}$. (c) Plot of upper critical field $\left(H_{c 2}\right)$ as a function of $T_{c}^{\text {mid }}$ for \#FM0, \#FM1, \#FM2 and \#FM3. The linear extrapolations to $\mathrm{T}=0 \mathrm{~K}$ are shown in the inset. 
Figure 2

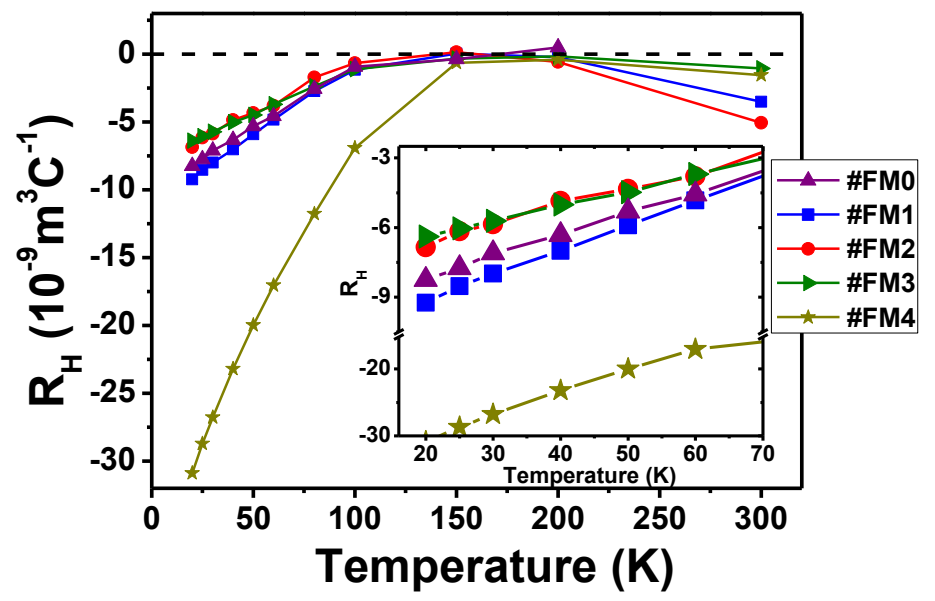

FIG 2. Hall coefficient $R_{H}$ as a function of temperature of five FeSe films. $R_{H}$ is determined as $R_{H}=\rho_{x y} / B$, dedicating the slope of hall transverse resistivity $\rho_{\mathrm{xy}}$ at designated field $\mathrm{B}$. Enlarged area from $20 \mathrm{~K}$ to $100 \mathrm{~K}$ is shown in the inset to distinguish the tendency clearly. 
Figure 3
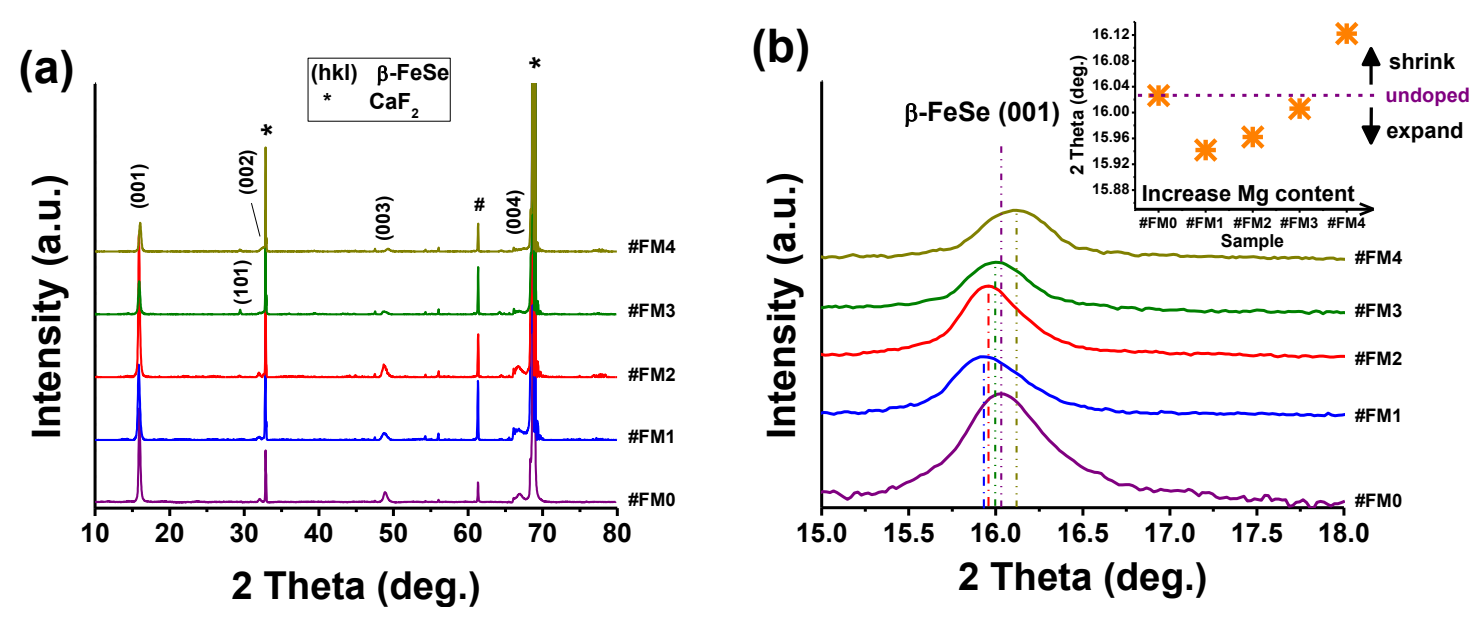

FIG. 3. (a) XRD $\theta-2 \theta$ patterns of five films grown on $\mathrm{CaF}_{2}$ (100) substrate. The spectrums are shown on a linear scale. Number signs (hkl) represent $\beta$-FeSe phases and the pound sign stands for the unidentified peak. (b) Magnified interval near $\beta$-FeSe (001) peak. The spectrums are shown on a logarithmic scale and the locations of peaks are marked by dashed lines. In the inset, the specific location of $\beta$-FeSe (001) peak is shown by extracting $2 \theta$ values from original spectrums. The dashed line represents the case of un-doped sample. 
Figure 4

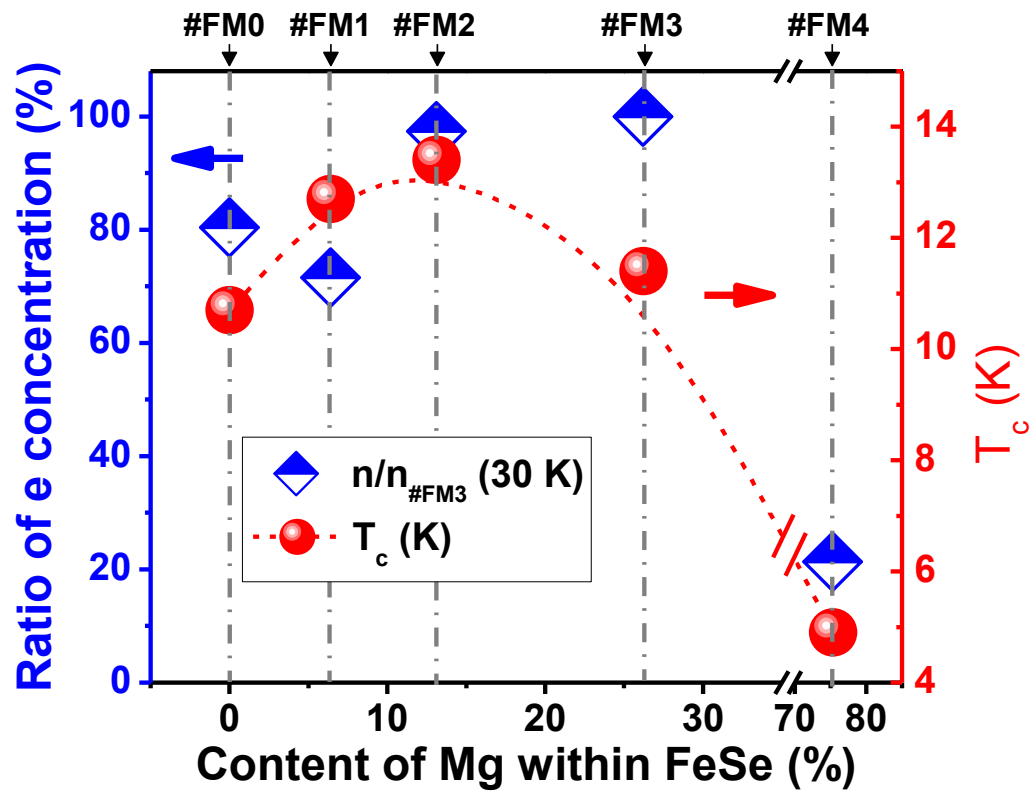

FIG. 4. The relative electron concentration $\mathrm{n} / \mathrm{n}_{\# \mathrm{FM} 3}$ at $30 \mathrm{~K}$ of all five samples is shown in the left $y$-axis and the tuning effect on $T_{c}$ by Mg-doping is shown in the right $y$-axis. The $x$-axis represents the content of $\mathrm{Mg}$ within FeSe films which is reflected by the atomic ratio of $\mathrm{Mg} / \mathrm{FeSe}$ detected by EDX characterization. The electron carrier concentration $n$ is calculated by $n=1 /\left(q \cdot R_{H}\right)$ and it is normalized by being divided by the $n_{\# F M 3}$ at $30 \mathrm{~K}$. The right $y$-axis exhibits $T_{c}$ values detected in different samples. A dome-shaped tendency is observed. 
Table of Contents (TOC) Graphic $(3.6 \mathrm{~cm} \times 4.83 \mathrm{~cm})$

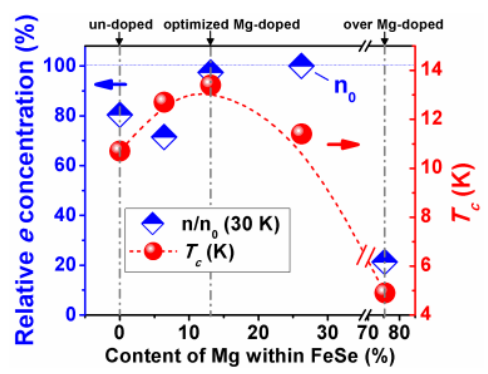

Superconducting transition temperature $\left(T_{c}\right)$ and relative electron concentration $(n)$ as a function of the content of Mg doping in FeSe films 